\title{
Cable-Stayed Architectures for Large Deployable Spacecraft
}

\author{
Andrew J. Lee* and Sergio Pellegrino ${ }^{\dagger}$ \\ California Institute of Technology, Pasadena, CA 91125
}

\begin{abstract}
Cable-stayed structural architectures, which use a combination of bending and axial loadcarrying modes, are potentially more efficient than structural architectures that rely only on bending. However, they are not widely used at present. In this paper, an analytical framework is established to compare the load carrying performance of cable-stayed vs. bending architectures by considering limiting conditions such as global buckling, local shell buckling, material failure, and excessive deflection. For structures of equal span, material properties, mass, and maximum deflection limit, the most efficient cable-stayed geometry is determined and its performance is compared to that of the beam. It is shown that the cable-stayed architecture is more efficient at withstanding external loads and remains optimal over the bending architecture. Design charts for optimal designs of cable-stayed structures for a range of lengths and loads are provided.
\end{abstract}

\section{Introduction}

Large deployable space structures are used for a variety of space missions that require large solar arrays [1], solar sails [2], sunshields [3], reflector antennas [4], and radar arrays [5]. These structures are required to be lightweight and to have high packaging efficiency. Due to stringent requirements on mass efficiency and surface accuracy, stiffness and stability against both internal and external loading are often the driving constraints of their structural designs. There have been many different structural concepts driven by technological advances in materials, manufacturing, and capabilities in analysis and testing, which can be categorized into structural architectures that are defined according to the way the loads are carried.

Many classical concepts for deployable structures are based on the bending architecture which relies on the flexural stiffness and strength derived from the depth of the structure. This architecture, schematically shown in Fig. 11a) for a deployable solar array, is composed of a series of composite sandwich plates covered with photovoltaic cells and connected by hinges that become locked after deployment. Many commercial satellites, as well as exploration missions such as Dawn [6] and Juno [7], have used solar arrays of this type.

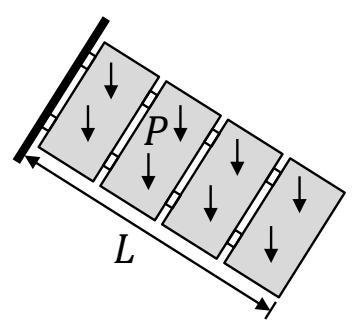

(a)

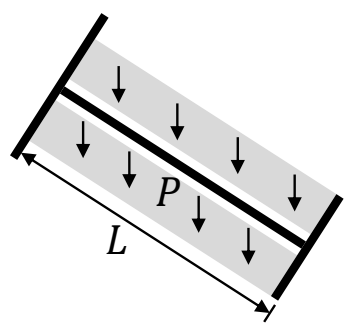

(b)

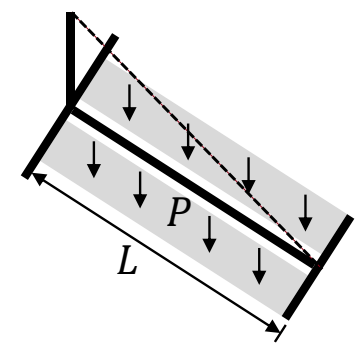

(c)

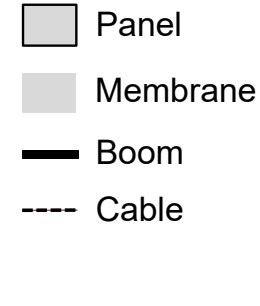

Membrane

Fig. 1 Deployable solar array concepts using (a) bending, (b) tensioned, and (c) cable-stayed architectures. $L$ is the length and $P$ is the solar radiation pressure.

The tensioned architecture is an alternative approach which aims to minimize the stowage volume required for large deployed surface areas. It is composed of a tensioned blanket attached to spreader bars at the ends, supported by a central deployable boom (as shown) or two deployable booms along the edges. The boom(s) are loaded in compression to react the tension in the blanket and the bending by the tip transverse load applied by the blanket. Flexible blanket solar arrays [8] are prominent examples of this architecture with many implementations having flight heritage, and square

\footnotetext{
* Postdoctoral Scholar, Graduate Aerospace Laboratories, 1200 E. California Blvd. MC 105-50. AIAA Member

$\dagger$ Joyce and Kent Kresa Professor of Aeronautics and Professor of Civil Engineering, Graduate Aerospace Laboratories, 1200 E. California Blvd. MC 105-50. AIAA Fellow.
} 
solar sails [9] with diagonal deployable booms adopt the same way of carrying loads. Fig. 11(b) shows an example flexible blanket solar array supported by a single boom.

The cable-stayed architecture is a variation to the bending and tensioned architectures that has received relatively small attention for spacecraft structures. Inspired by cable-stayed bridges, this architecture has an additional vertical column at the root which shares the effective loading with horizontal structural members through connected cables under tension. Fig. 11.c) shows a cable-stayed concept based on a modification of the example in Fig. 1.b).

Stayed columns have been shown to be highly mass efficient when the dimensionless structural index $P_{c r} / E L^{2}$ (where $P_{c r}$ is the critical axial buckling load, $E$ is the elastic modulus, and $L$ is the length) is small [10], which is the case for large mass-minimized space structures [11]. Hedgepeth [12] first conceptualized a large space structure in the form of a solar reflector spacecraft that utilize stayed columns. Belvin [13] characterized the linear vibration and buckling characteristics of a pretensioned stayed column for hoop-column antennas. More recently, Jones et. al [14] showed through nonlinear finite element analysis that cable stays connected to a vertical column can be used to increase the stiffness of a tensioned architecture implementation of a large aperture radar antenna. The cable stays can negate large bending deformation by increasing the lateral stiffness of the system while lowering the membrane surface tension required to match the fundamental frequency of an equivalent bending architecture. These findings were specific to the DARPA Innovative Space-Based Radar Antenna Technology [15] platform's design requirements, but this brief review has highlighted the potential for harnessing the potential of this type of architecture for a wide range of applications. The potential performance improvements of cable-stayed architectures may offset the added complexity in packaging and deployment.

This paper analyzes the load carrying capability of the cable-stayed architecture relative to a comparable bending architecture for large spacecraft under pressure loading. It is assumed that other forms of loading such as inertial forces, gravity gradient, etc. can be considered as equivalent pressure. To allow a comparative design study based on analytical solutions, the two architectures are simplified to one-dimensional structures with length $L$ and distributed load $p$, as shown in Fig. 22 The structures are assumed symmetric with respect to the $z$-axis. The bending architecture is analyzed as a cantilevered Euler-Bernoulli beam while the cable-stayed architecture is a horizontal beam-column connected at the tip to a vertical column by a single, taut cable. This particular composition of the cable-stayed architecture is chosen

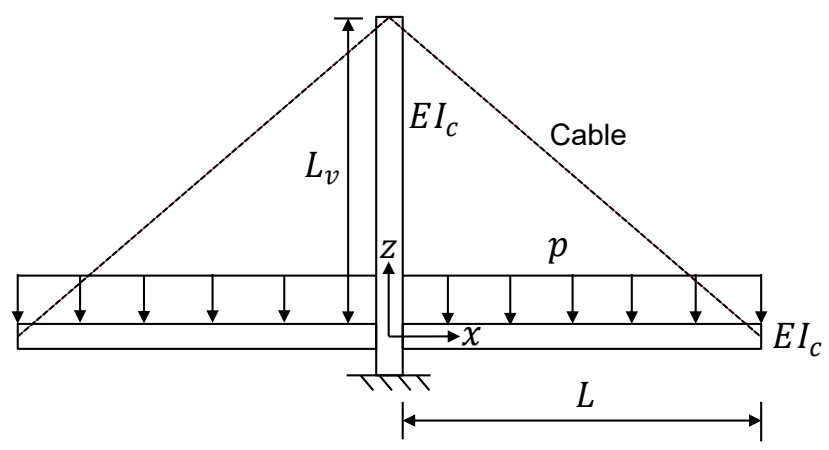

(a)

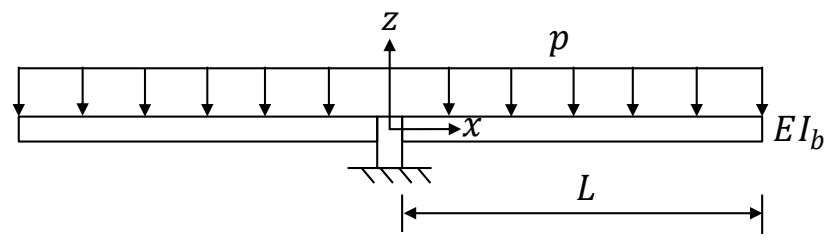

(b)

Fig. 2 (a) The cable-stayed architecture consists of horizontal beam-columns connected to a vertical column of length $L_{v}$ through cables (dashed line) attached to the free ends. (b) The bending architecture consists of cantilevered Euler-Bernoulli beams. The horizontal beam-column and the beam have length $L$ and are under distributed loading $p$. 
because it is being compared to the bending architecture instead of the tensioned architecture. $E$ is the elastic modulus and $I_{b}, I_{c}$ are the second moment of areas of the bending and cable-stayed architectures, respectively. Throughout the paper, the subscript $b$ denotes the bending architecture and the subscript $c$ denotes the cable-stayed architecture.

The aim of the present study is to compare the cable-stayed and bending architectures by considering the loads that induce global buckling, local buckling, material failure, and excessive deflection. For each architecture, the smallest load among these four limiting conditions characterizes the performance of each specific design. The architectures' geometric parameters are varied while they share the same material properties to maintain identical mass and maximum deflection (equivalent to having equal stiffness) throughout the comparisons. A deflection limit is imposed to deal with design cases where buckling or material failure occurs at disproportionately large deflections.

The paper is laid out as follows. Section $\mathrm{II}$ derives the distributed loads at buckling, material failure, and excessive deflection for the cable-stayed and bending architectures along with the analytical conditions for identical mass and maximum deflection. Section III presents the comparison procedure for identifying the superior architecture and a comparative design study is done for a range of lengths. Then Section IV characterizes the optimal designs from the design study based on the maximum load carrying performance.

\section{Analytical Framework}

This section determines the loads that correspond to the different design limiting conditions. The horizontal beam-column and the vertical column in the cable-stayed architecture are assumed to be thin-walled circular tubes with equal cross-sectional radius $r_{c}$ and thickness $t_{c}$. The cantilevered beam in the bending architecture is also assumed to be a thin-walled circular tube, with radius $r_{b}$ and thickness $t_{b}$ that are in general different from the properties of the cable-stayed architecture. It is assumed that the transverse distributed load $p$ is the only external loading and the deflections are small. Both the tubes and the cable are assumed to be inextensible.

\section{A. Global Euler Buckling}

The loads in the cable-stayed architecture must first be found before deriving the global Euler buckling loads of both members in compression. Starting with the transversely loaded horizontal beam-column in Fig. 3. a), the inextensible cable acts as a vertical constraint at the tip $(x=L)$, which is equivalent to a roller boundary condition. For symmetry, the root of the beam-column is modeled as clamped.

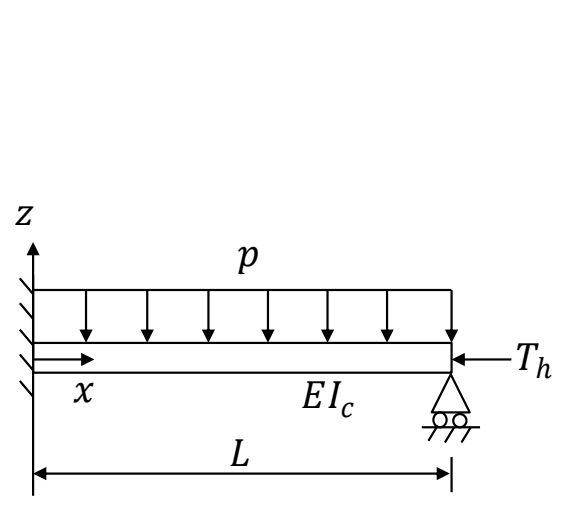

(a)

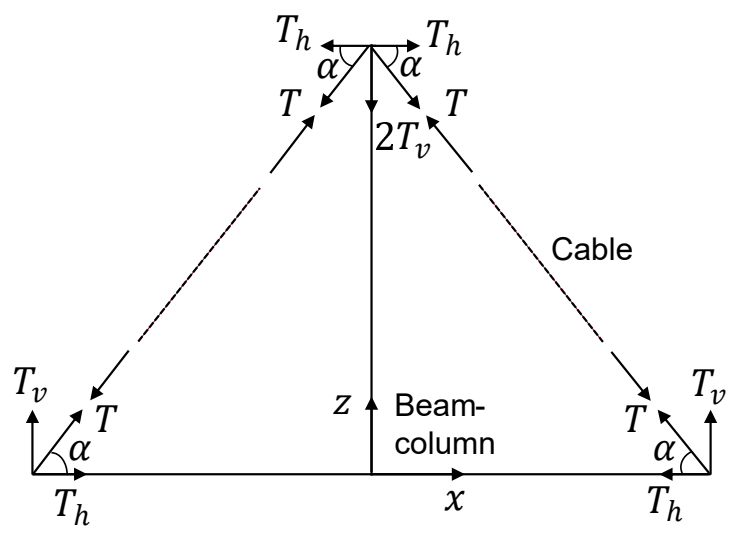

(b)

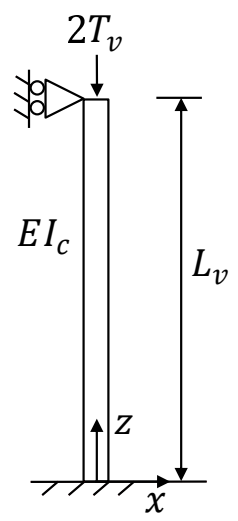

(c)

Fig. 3 Cable-stayed architecture parameters, boundary conditions, and reaction forces from distributed loading $p$. (a) Horizontal beam-column, (b) inextensible cable, and (c) vertical column.

The tip-supported beam-column is one times statically indeterminate, and the vertical component of the tip reaction, $T_{v}$, is given by

$$
T_{v}=\frac{3}{8} p L
$$

This reaction provides the vertical component of the cable tension. Then the cable tension $T$ can be found from 
vertical equilibrium and then the horizontal component $T_{h}$ provides the axial compression in the beam-column:

$$
T_{h}=\frac{3}{8} p L \cot \alpha
$$

where $\alpha$ is the cable angle and is given by:

$$
\alpha=\arctan \frac{L_{v}}{L}
$$

Substituting Eq. 3 into Eq. 2 yields:

$$
T_{h}=\frac{3 p L^{2}}{8 L_{v}}
$$

Due to mirror symmetry about the $z$-axis, the horizontal components of the cable tensions $T_{h}$ at the tip of the vertical column, Fig. 3(c), are in equilibrium and hence no tip shear force is applied to the column. The vertical components are in equilibrium with an axial compression $2 T_{v}$. To analyze the global buckling of the vertical column, a horizontal roller boundary condition at the tip is assumed, since the cables act as constraints in the $x$-direction. The column is assumed to be clamped at the root. If out-of-plane buckling is considered, then the equivalent boundary condition would be a free end at $z=L_{v}$, but out-of-plane behavior is not considered in this paper.

Having defined the loads on both the horizontal beam-column and the vertical column as a function of $p$, the critical value for global buckling can be found. Setting the Euler buckling load for the fixed-pinned vertical column [16] equal to the axial compression in the column gives:

$$
T_{v_{c r}}=1.023 \pi^{2} \frac{E I_{c}}{L_{v}^{2}}
$$

and, similarly, setting the Euler buckling load for the fixed-pinned horizontal beam-column equal to the axial compression in the beam-column gives:

$$
T_{h_{c r}}=2.045 \pi^{2} \frac{E I_{c}}{L^{2}}
$$

where $I_{c}$ is the second moment of area for the vertical column and horizontal beam-column for bending in the $x z$ plane. For the chosen thin-walled tubes, $I_{c}=\pi t_{c} r_{c}^{3}$.

Substituting $T_{v}$ and $T_{h}$ in Eqs. 1 and 4 into the Euler buckling loads in Eqs. 5 and 6 and solving for $p$ provides the critical values of $p$ corresponding to global buckling of the vertical column, $p_{c v_{g c r}}$, and the horizontal beam-column, $p_{c h_{g c r}}$. They have the following expressions:

$$
\begin{gathered}
p_{c v_{g c r}}=2.727 \pi^{3} \frac{E t_{c} r_{c}^{3}}{L^{3} \hat{L}_{v}^{2}} \\
p_{c h_{g c r}}=5.453 \pi^{3} \frac{E t_{c} r_{c}^{3} \hat{L}_{v}}{L^{3}}
\end{gathered}
$$

where $L_{v}$ has been non-dimensionalized as $\hat{L}_{v}=\frac{L_{v}}{L}$. Note that the subscript $g$ denotes global buckling.

In the bending architecture there is no axial compression for the beam, and hence in this case there is no global buckling limit.

\section{B. Local Shell Buckling}

For both architectures, local shell buckling occurs when the maximum compressive stress in the thin-walled circular tube reaches the critical buckling stress $\sigma_{c r}$ of a cylindrical shell:

$$
\sigma_{c r}=\gamma_{b, c} \sigma_{c l}
$$

where $\sigma_{c l}$ is the critical buckling stress for a geometrically perfect cylindrical shell and $\gamma_{b, c}$ denotes the knockdown factor that accounts for the buckling load reduction due to imperfections. $\sigma_{c l}$ has the expression [17]:

$$
\sigma_{c l}=\frac{E t}{r \sqrt{3\left(1-v^{2}\right)}}
$$


where $v$ is the Poisson's ratio, $r$ is the tube radius, and $t$ is the tube thickness of either architecture.

The knockdown factors for a tube under pure bending, $\gamma_{b}$, and under pure axial compression, $\gamma_{c}$, are based on the empirical curves in NASA SP-8007 [18] and are a function of the radius to thickness ratio $r / t$ :

$$
\begin{aligned}
& \gamma_{b}=1-0.731\left(1-e^{\frac{-1}{16} \sqrt{\frac{r}{t}}}\right) \\
& \gamma_{c}=1-0.901\left(1-e^{\frac{-1}{16} \sqrt{\frac{r}{t}}}\right)
\end{aligned}
$$

For the cable-stayed architecture's horizontal beam-column, the maximum stress $\sigma_{c h}$ is the sum of the bending and axial stresses due to $p$ and $T_{h}$, respectively, and is given by:

$$
\sigma_{c h}=\frac{M_{c h_{\max }}}{\pi r_{c}^{2} t_{c}}+\frac{T_{h}}{2 \pi r_{c} t_{c}}
$$

The maximum bending moment is conservatively estimated by adding the bending moment at the root to the maximum moment due to the offset of the axial compression $T_{h}$ that comes from the maximum deflection of the beam-column $w_{c h_{\max }}$ (derived later in Eq. 26):

$$
M_{c h_{\max }}=\frac{p L^{2}}{8}+T_{h}\left|w_{c h_{\max }}\right|
$$

The shell buckling stress for combined bending and compression is obtained from the interaction equation [18] which incorporates both the bending and compressive knockdown factors:

$$
\sigma_{c l}=\frac{1}{\gamma_{b}}\left(\frac{M_{c h_{\max }}}{\pi r_{c}^{2} t_{c}}\right)+\frac{1}{\gamma_{c}}\left(\frac{T_{h}}{2 \pi r_{c} t_{c}}\right)
$$

Substituting Eqs. 4, 11, 12, and 14 into Eq. 15 and solving for $p$ results in the critical load $p_{c h_{l c r}}$ that induces local shell buckling in the horizontal beam-column:

$$
\begin{aligned}
p_{c h_{l c r}} & =\frac{4}{3 L^{4}\left(3 \gamma_{b} r_{c}-\gamma_{c} L \hat{L}_{v}(0.855 a-2)\right)}\left[2 a E \gamma_{c} L^{2} \hat{L}_{v}^{2} \pi^{3} r_{c}^{3} t_{c}+3 a E \gamma_{b} L \hat{L}_{v} \pi^{3} r_{c}^{4} t_{c}\right. \\
& +6 \gamma_{b} \gamma_{c} L^{3} \hat{L}_{v} \pi r_{c}^{2} t_{c} \sigma_{c l}-\left[L ^ { 2 } \hat { L } _ { v } ^ { 2 } \pi ^ { 2 } r _ { c } ^ { 4 } t _ { c } ^ { 2 } \left(24 a E \gamma_{b} \gamma_{c} L^{2} \pi^{2} r_{c} \sigma_{c l}\left(0.855 a-3 \gamma_{b} r_{c}-2\right)\right.\right. \\
& \left.\left.\left.+\left(a E \pi^{2} r_{c}\left(2 \gamma_{c} L \hat{L}_{v}+3 \gamma_{b} r_{c}\right)+6 \gamma_{b} \gamma_{c} L^{2} \sigma_{c l}\right)^{2}\right)\right]^{\frac{1}{2}}\right]
\end{aligned}
$$

where the coefficient $a=2.045$ and the subscript $l$ denotes local buckling.

The vertical column is loaded in pure compression, with magnitude $2 T_{v}$ and hence the maximum stress is:

$$
\sigma_{c v}=\frac{T_{v}}{\pi r_{c} t_{c}}
$$

After substituting Eq. 1 into Eq. 17, then equating it to $\sigma_{c r}$ in Eq. 9, and then solving for $p$ yields the critical load $p_{c v_{l c r}}$ for local shell buckling of the vertical tube:

$$
p_{c v_{l c r}}=\frac{8 \pi \gamma_{c} E t_{c}^{2}}{3 L \sqrt{3\left(1-v^{2}\right)}}
$$

In the case of the bending architecture, the maximum bending moment is $M_{b}(0)=\frac{p L^{2}}{2}$ and the resulting maximum stress is:

$$
\sigma_{b}=\frac{p L^{2}}{2 \pi r_{b}^{2} t_{b}}
$$

The critical load that corresponds to local buckling of the cantilevered beam is obtained by setting the maximum bending stress $\sigma_{b}$ equal to $\sigma_{c r}$ in Eq. 9 Then, solving for $p$ results in the critical load $p_{b_{l c r}}$ :

$$
p_{b_{l c r}}=\frac{2 \pi \gamma_{b} E r_{b} t_{b}^{2}}{L^{2} \sqrt{3\left(1-v^{2}\right)}}
$$

This mode of failure is characterized by local buckling at the lowest point of the tube's root, corresponding to the maximum compressive stress. 


\section{Material Failure}

The failure stress $\sigma_{y}$ can be expressed simply as a function of the elastic modulus $E$ and the failure strain $\epsilon_{y}$ :

$$
\sigma_{y}=E \epsilon_{y}
$$

Material failure will occur at the points of highest stress in the tubes' cross-section, when the maximum stresses reach the failure stress. For the cable-stayed architecture, equating $\sigma_{c h}$ and $\sigma_{c v}$ in Eqs. 13 and 17 to $\sigma_{y}$ in Eq. 21 and solving for $p$ provides the loads $p_{c h_{y}}$ and $p_{c v_{y}}$ that cause material failure for the horizontal beam-column and the vertical column, respectively:

$$
\begin{gathered}
p_{c h_{y}}=\frac{4}{3 L^{4}\left(3 r_{c}-L \hat{L}_{v}(0.855 a-2)\right)}\left[2 a E L^{2} \hat{L}_{v}^{2} \pi^{3} r_{c}^{3} t_{c}+3 a E L \hat{L}_{v} \pi^{3} r_{c}^{4} t_{c}+6 L^{3} \hat{L}_{v} \pi r_{c}^{2} t_{c} \sigma_{y}\right. \\
\left.-\left[L^{2} \hat{L}_{v}^{2} \pi^{2} r_{c}^{4} t_{c}^{2}\left(24 a E L^{2} \pi^{2} r_{c} \sigma_{y}\left(0.855 a-3 r_{c}-2\right)+\left(a E \pi^{2} r_{c}\left(2 L \hat{L}_{v}+3 r_{c}\right)+6 L^{2} \sigma_{y}\right)^{2}\right)\right]^{\frac{1}{2}}\right] \\
p_{c v_{y}}=\frac{8 \pi E t_{c} r_{c} \epsilon_{y}}{3 L}
\end{gathered}
$$

For the bending architecture, equating $\sigma_{b}$ in Eq. 19 to $\sigma_{y}$ in Eq. 21 and solving for $p$ provides the load $p_{b_{y}}$ that causes material failure for the beam:

$$
p_{b_{y}}=\frac{2 \pi E r_{b}^{2} t_{b} \epsilon_{y}}{L^{2}}
$$

\section{Excessive Deflection}

The last limiting condition for both architectures is excessive deflection. Given the fixed-pinned boundary conditions of the cable-stayed architecture's horizontal beam-column in Fig. 3.a), the transverse deflection $w_{c h}$ due to the distributed load $p$ and the axial compression $T_{h}$ is given by:

$$
w_{c h}(x)=\left(\frac{1}{1-T_{h} / T_{h_{c r}}}\right)\left[\frac{-p x^{2}}{48 E I_{c}}\left(2 x^{2}-5 L x+3 L^{2}\right)\right]
$$

where $T_{h}$ and $T_{h_{c r}}$ are given by Eqs. 4 and 6 , respectively. Note the amplification factor $\frac{1}{1-T_{h} / T_{h_{c r}}}$ that accounts for the additional deflection caused by the axial compression. This expression of the deflection provides a simple and rather accurate approximation of the exact beam-column deflection, especially when $T_{h}<<T_{h_{c r}}$ [16].

The maximum deflection $w_{c h_{\max }}$ occurs at $x=0.578 \mathrm{~L}$ and has the expression:

$$
w_{c h_{\max }}=\frac{-0.428 a \hat{L}_{v} L^{5} p}{8 a \pi^{2} E I_{c} \hat{L}_{v} L-3 p L^{4}}
$$

where again $a=2.045$. The vertical column is only axially loaded and does not bend.

The load that causes excessive deflection for the cable-stayed architecture is found by equating Eq.26/to the deflection limit $w_{d l}$ and solving for $p$ :

$$
p_{c h_{d l}}=\frac{184.6 a E I_{h} \hat{L}_{v} \hat{w}_{d l}}{L^{3}\left(a \hat{L}_{v}+7.015 \hat{w}_{d l}\right)}
$$

where $w_{d l}$ has been non-dimensionalized as $\hat{w}_{d l}=\frac{w_{d l}}{L}$.

For the bending architecture the transverse deflection $w_{b}$ is given by:

$$
w_{b}(x)=\frac{-p x^{2}}{24 E I_{b}}\left(x^{2}-4 L x+6 L^{2}\right)
$$

where the maximum displacement $w_{b_{\max }}$ is at the tip $(x=L)$ and is given by:

$$
w_{b_{\max }}=-\frac{p L^{4}}{8 E I_{b}}
$$

Then, following the same procedure as for the cable-stayed architecture, the load that causes excessive deflection is found to be:

$$
p_{b_{d l}}=\frac{8 E I_{b} \hat{w}_{d l}}{L^{3}}
$$




\section{E. Conditions for Equal Maximum Deflection and Mass}

The condition for identical maximum deflections between the two architectures is obtained by equating Eqs. 26 and 29 substituting $I=\pi t r^{3}$ for each architecture, and simplifying. The resulting expression is:

$$
t_{c} r_{c}^{3}-0.0433 t_{b} r_{b}^{3}=\frac{0.0380 p L^{3}}{a \pi E \hat{L}_{v}}
$$

This equation shows that if the elastic modulus $E$ and lengths $L$ and $\hat{L}_{v}$ are held constant and the loading is varied, then the radius or thickness of either architecture must be varied to maintain identical maximum deflections.

Eq. 31 can be expressed as a function of the non-dimensionalized deflection limit $\hat{w}_{d l}$ by substituting Eq. 27 for $p$, which results in:

$$
\frac{r_{c}}{r_{b}}=0.351\left[\frac{t_{b}}{t_{c}}\left(\frac{a \hat{L}_{v}+6.993 \hat{w}_{d l}}{a \hat{L}_{v}}\right)\right]^{\frac{1}{3}}
$$

This equation provides a constraint between the bending and cable-stayed architectures such that they have equal maximum deflections when the deflection limit is reached.

The total masses $m_{c}$ and $m_{b}$ of the cable-stayed and bending architectures are respectively:

$$
\begin{gathered}
m_{c}=2 \pi r_{c} t_{c} \rho L\left(1+\frac{\hat{L}_{v}}{2}\right)\left(1+\frac{m_{\text {cable }}}{m_{h v}}\right) \\
m_{b}=2 \pi r_{b} t_{b} \rho L
\end{gathered}
$$

where $\rho$ is the density for both architectures, $m_{\text {cable }}$ is the mass of the cable, and $m_{h v}$ is the combined mass of the horizontal beam-column and vertical column. Note that both $m_{b}$ and $m_{c}$ are for half of the complete architectures shown in Fig. 2

Then the condition for equal mass between the two architectures is found by equating Eqs. 33 and 34 , which gives:

$$
\frac{r_{b}}{r_{c}}=\frac{t_{c}}{t_{b}}\left(1+\frac{\hat{L}_{v}}{2}\right)\left(1+\frac{m_{\text {cable }}}{m_{h v}}\right)
$$

\section{Comparison of Architectures}

The distributed loads corresponding to the limiting conditions of buckling, material failure, and excessive deflection for the two architectures are compared while maintaining equal mass and maximum deflection. The notations for these loads are shown in Table 1 for reference. The material properties are assumed to be $E=70 \mathrm{GPa}, v=0.3, \rho=1600$ $\mathrm{kg} / \mathrm{m}^{3}$, and $\epsilon_{y}=0.01$. For the cable-stayed architecture, the cable mass is assumed to be one tenth of the combined mass of the horizontal beam-column and the vertical column, i.e. $\frac{m_{\text {cable }}}{m_{h v}}=0.1$.

Table 1 Notations for distributed loads corresponding to design limiting conditions.

\begin{tabular}{cccc}
\hline & $\begin{array}{c}\text { Cable-stayed: } \\
\text { Vertical Column }\end{array}$ & $\begin{array}{c}\text { Cable-stayed: } \\
\text { Horizontal Beam-column }\end{array}$ & $\begin{array}{c}\text { Bending: } \\
\text { Beam }\end{array}$ \\
\hline Global Euler Buckling & $p_{c v_{g c r}}$ & $p_{c h_{g c r}}$ & N/A \\
Local Shell Buckling & $p_{c v_{l c r}}$ & $p_{c h_{l c r}}$ & $p_{b_{l c r}}$ \\
Material Failure & $p_{c v_{y}}$ & $p_{c h_{y}}$ & $p_{b_{y}}$ \\
Excessive Deflection & N/A & $p_{c h_{d l}}$ & $p_{b_{d l}}$ \\
\hline
\end{tabular}

\section{A. Procedure}

The first step in the comparison of the two architectures is to rearrange the mass equation of the cable-stayed architecture in Eq. 33 so that it is expressed in terms of the thickness to radius ratio $\frac{t_{c}}{r_{c}}$ and linear mass density $\frac{m_{c}}{L}$ :

$$
t_{c}=\sqrt{\frac{\left(\frac{m_{c}}{L}\right)\left(\frac{t_{c}}{r_{c}}\right)}{2 \pi \rho\left(1+\frac{\hat{L}_{v}}{2}\right)\left(1+\frac{m_{\text {cable }}}{m_{h v}}\right)}}
$$


The thickness to radius ratio is chosen as $\frac{t_{c}}{r_{c}}=0.0075$ and the linear mass density as $\frac{m_{c}}{L}=0.1 \mathrm{~kg} / \mathrm{m}$. These are both reasonable values for load carrying tubular booms [9]. The only remaining free parameter is the non-dimensional length of the vertical-column $\hat{L}_{v}$ and this is chosen as an independent variable.

The four unknowns $t_{c}, t_{b}, r_{c}$, and $r_{b}$ are obtained from Eqs. 32, 35, 36 with the specified $\frac{t_{c}}{r_{c}}$ ratio. These cross-sectional parameters ensure that the equal maximum deflection and mass conditions are satisfied for any given $\hat{L}_{v}$. The non-dimensionalized deflection limit is set to $\hat{w}_{d l}=0.1$. To maintain equal mass, the cross-sectional area of the cable-stayed architecture will always be smaller than the bending architecture to account for the greater total length of horizontal beam-column plus the vertical column.

Having defined all of the necessary geometric and material parameters, the load limits are computed as a function of $\hat{L}_{v}$ for global buckling (Eqs. 7 and 8, , local shell buckling (Eqs. 16, 18, and 20, material failure (Eqs. 22,-24), and excessive deflection (Eqs. 27 and 30). They have been plotted for cable-stayed and bending architectures with $L=20 \mathrm{~m}$ and $m_{b, c}=2 \mathrm{~kg}$ in Fig. 4 (a) and (b), respectively.
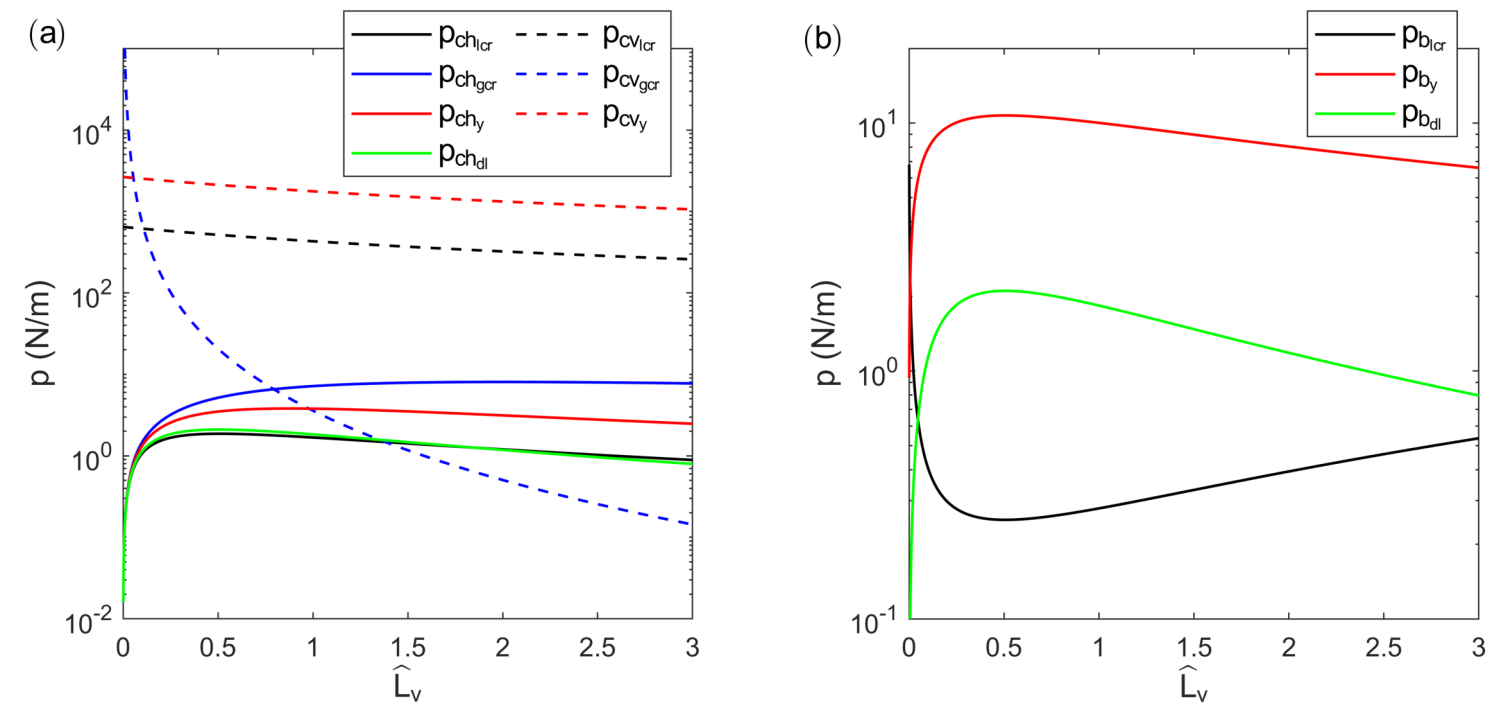

Fig. 4 Loads that cause global buckling, local shell buckling, material failure, and excessive deflection as a function of $\hat{L}_{v}$ for (a) cable-stayed architecture and (b) bending architecture. The mass is $m_{b, c}=2 \mathrm{~kg}$ and the span is $L=20 \mathrm{~m}$. The actual load limit is the lowest envelope of the plotted curves.

Fig. 4(a) shows that the (non-dimensional) vertical column length $\hat{L}_{v}$ in the cable-stayed architecture has a significant effect on the maximum load that can be carried by this architecture, which is defined by the lowest envelope of the plotted curves. For $\hat{L}_{v}<0.794$ the horizontal beam-column buckles globally for much lower loads than the vertical column, since the former is relatively longer than the latter while sharing the same cross-sectional geometry and material. For $\hat{L}_{v}>0.794$, the vertical column buckle globally first, as seen by the sharp decrease in $p_{c v_{g c r}}$ under $p_{c h_{g c r}}$. For both local shell buckling and material failure, the horizontal beam-column will always fail first due to its higher maximum stress being attributed to both axial compression from the cable reaction and bending from the distributed load. Fig. 4 b) shows that for the bending architecture the load limit is local shell buckling for $0.047 \leq \hat{L}_{v} \leq 3$ and excessive deflection for $\hat{L}_{v}<0.047$. The reason why the load limits vary with $\hat{L}_{v}$ is because the radius $r_{b}$ and thickness $t_{b}$ are both related to $\hat{L}_{v}$ by the constraints of equal mass and maximum deflection between the two architectures.

Defining the load limits of each architecture, $p_{c_{\text {lim }}}$ and $p_{b_{\text {lim }}}$, as the smallest among the loads corresponding to global buckling, local shell buckling, material failure, and excessive deflection:

$$
\begin{gathered}
p_{c_{l i m}}=\min \left\{p_{c h_{g c r}}, p_{c v_{g c r}}, p_{c h_{l c r}}, p_{c v_{l c r}}, p_{c h_{y}}, p_{c v_{y}}, p_{c h_{d l}}\right\} \\
p_{b_{l i m}}=\min \left\{p_{b_{l c r}}, p_{b_{y}}, p_{b_{d l}}\right\}
\end{gathered}
$$

allows a performance comparison between the two architectures. Fig. 5 shows a plot of $p_{c_{\text {lim }}}$ and $p_{b_{\text {lim }}}$ where the cable-stayed architecture exhibits two different failure modes: local shell buckling $p_{c h_{l c r}}$ of the horizontal beam-column 
for $0 \leq \hat{L}_{v} \leq 1.383$ and global buckling $p_{c v_{g c r}}$ of the vertical column for $\hat{L}_{v}>1.383$. It outperforms the bending architecture for $0.048 \leq \hat{L}_{v} \leq 2.139$ while the bending architecture is better outside of this $\hat{L}_{v}$ range. This result indicates that the bending architecture would overtake the cable-stayed architecture for designs with overly long columns $\left(\hat{L}_{v}<2.139\right)$, which of course should be avoided.

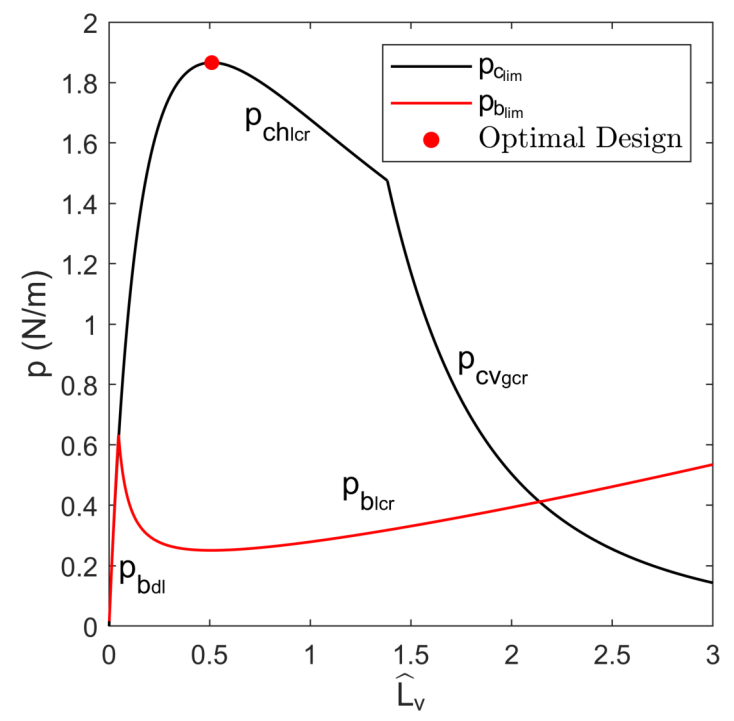

Fig. 5 Load limits for the cable-stayed architecture $p_{c_{l i m}}$ (black line) and the bending architecture $p_{b_{\text {lim }}}$ (red line) with identical mass of $m_{b, c}=2 \mathrm{~kg}$, maximum deflection at $\hat{w}_{d l}=0.1$, and $L=20 \mathrm{~m}$. The labels for each region of the plot identify which specific load limit is active.

An important result shown in Fig. 5 is the design that can carry the largest load which is $p=1.866 \mathrm{~N} / \mathrm{m}$. It is obtained with the cable-stayed architecture for $\hat{L}_{v}=0.510$, i.e. $L_{v}=10.2 \mathrm{~m}$. It will fail by local shell buckling in the horizontal beam-column. Specifically, this design has the radius $r_{c}=31.0 \mathrm{~mm}$, thickness $t_{c}=0.233 \mathrm{~mm}$, and maximum deflection of $w_{c h_{\max }}=-1.65 \mathrm{~m}$, corresponding to $\left|\frac{w_{c h_{\max }}}{L}\right|=0.083$. The deflected shape of this particular cable-stayed design under the maximum load is shown in Fig. 6

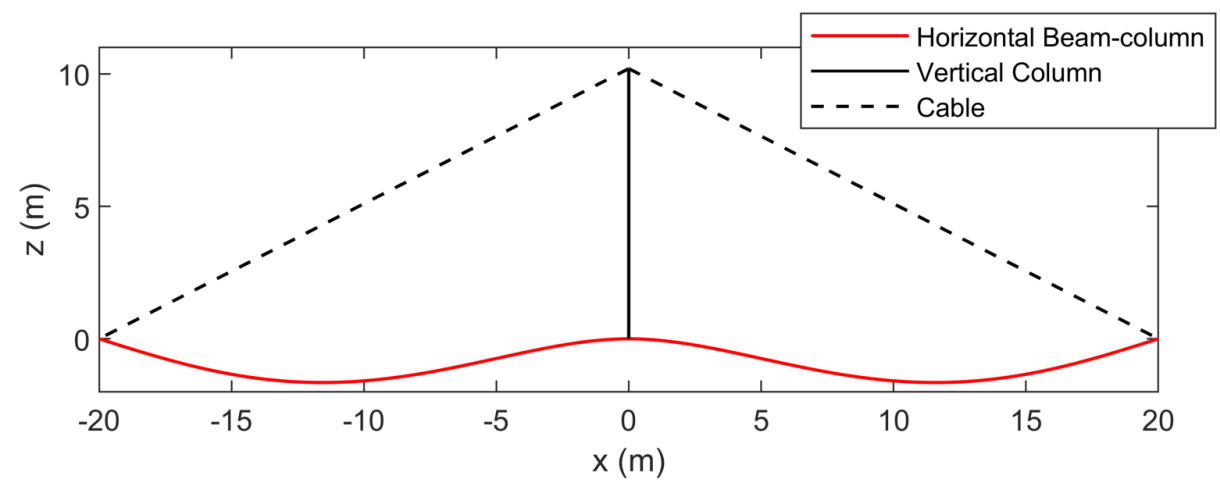

Fig. 6 Deflected shape of optimal design for $L=20 \mathrm{~m}$ and $m_{c}=2 \mathrm{~kg}$. It has $L_{v}=10.2 \mathrm{~m}, r_{c}=31.0 \mathrm{~mm}$, $t_{c}=0.233 \mathbf{m m}$, and can carry a load of $1.866 \mathrm{~N} / \mathbf{m}$ with a maximum deflection of $-1.65 \mathbf{m}$. 


\section{B. Comparison for Additional Lengths}

The procedure outlined above selects the best architecture and the corresponding optimal design. It has been applied to a range of different lengths and masses while maintaining the same material properties and thickness to radius ratio $\frac{t_{c}}{r_{c}}=0.0075$. The results are shown in Fig. 7 for $5 \leq L \leq 100 \mathrm{~m}$ and $0 \leq \hat{L}_{v} \leq 3$ for two linear mass densities $\frac{m_{b, c}}{L}=0.1$ $\mathrm{kg} / \mathrm{m}$, Fig. 7 (a), and $\frac{m_{b, c}}{L}=1 \mathrm{~kg} / \mathrm{m}$, Fig. 7 b). These plots identify regions of the design space, separated by black lines, where a different load limit dominates. The load limits are computed from $\max \left\{p_{c_{\text {lim }}}, p_{b_{\text {lim }}}\right\}$. Note that there exist regions where $p_{c h_{d l}}=p_{b_{d l}}$ due to both architectures having excessive deflection and being limited by $\hat{w}_{d l}=0.1$.
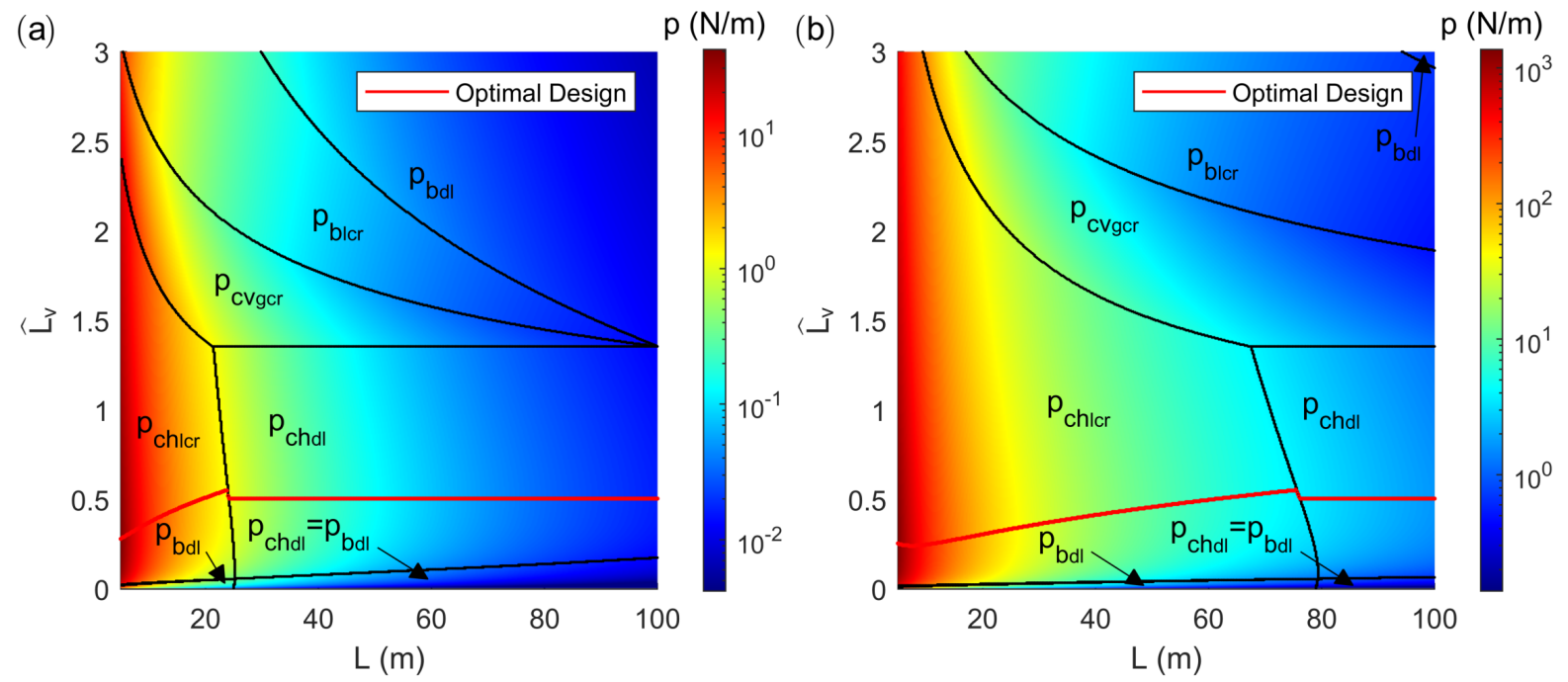

Fig. 7 Maximum load that can be carried by the two architectures for (a) $\frac{m_{b, c}}{L}=0.1 \mathrm{~kg} / \mathbf{m}$ and (b) $\frac{m_{b, c}}{L}=1$ $\mathrm{kg} / \mathrm{m}$. The specific limiting condition is labelled for each region and the optimal designs as a function of $L$ are shown by the red line.

For any given length, $\hat{L}_{v}$ ranges exist which favor the bending architecture where its designs are limited by excessive deflection and local shell buckling, particularly at low and high values of $\hat{L}_{v}$. For both linear mass densities however, the optimal designs shown by the red lines always correspond to the cable-stayed architecture due to retaining the maximum limiting load across the $\hat{L}_{v}$ range for each $L$. At $\frac{m_{b, c}}{L}=0.1 \mathrm{~kg} / \mathrm{m}$, the optimal cable-stayed designs are limited by local shell buckling of the horizontal beam-column for $L<23.8 \mathrm{~m}$ and excessive deflection for $L>24.2 \mathrm{~m}$ while the inbetween lengths are simultaneously limited by both conditions $\left(p_{c h_{l c r}}=p_{c h_{d l}}\right)$. As the length increases, the corresponding optimal $\hat{L}_{v}$ initially increases from 0.28 to 0.56 , then settles at 0.505 once the designs are deflection limited. For any $\hat{L}_{v}$, the design limiting load also decreases as $L$ is raised due to the constant $\frac{m_{b, c}}{L}$ keeping the cross-sectional radii and thicknesses fixed for both architectures. If the mass is significantly increased per length with $\frac{m_{b, c}}{L}=1 \mathrm{~kg} / \mathrm{m}$, the transition lengths where the optimal cable-stayed designs become limited by both deflection and local shell buckling of the horizontal beam-column are increased to $75.4 \leq L \leq 76.2 \mathrm{~m}$. This increase indicates that heavier cable-stayed designs are less susceptible to large deflections and will buckle earlier due to the larger cross-sectional radii and thicknesses. More importantly, the additional mass will allow the structures to have larger design limiting loads for the same lengths.

\section{Optimal Designs}

To more systematically characterize the optimal designs, their maximum load limit $\max \left\{p_{c_{\text {lim }}}, p_{b_{\text {lim }}}\right\}$, vertical column length $\hat{L}_{v}$, and total mass $m_{b, c}$ have been plotted as functions of $L$ and $\frac{m_{b, c}}{L}$ in Figs. 8 and 9 . The material properties and initial geometric parameters are the same as in the previous analysis. In these figures, solid lines mark the transitions between different limiting conditions, dashed lines mark the order-of-magnitude variations of $\max \left\{p_{c_{l i m}}, p_{b_{l i m}}\right\}$, and the dotted lines mark variations of total mass. Finally, any optimal designs with $t_{b, c}<0.1 \mathrm{~mm}$ have been removed from the figures to account for practical limitations on tube thickness. 

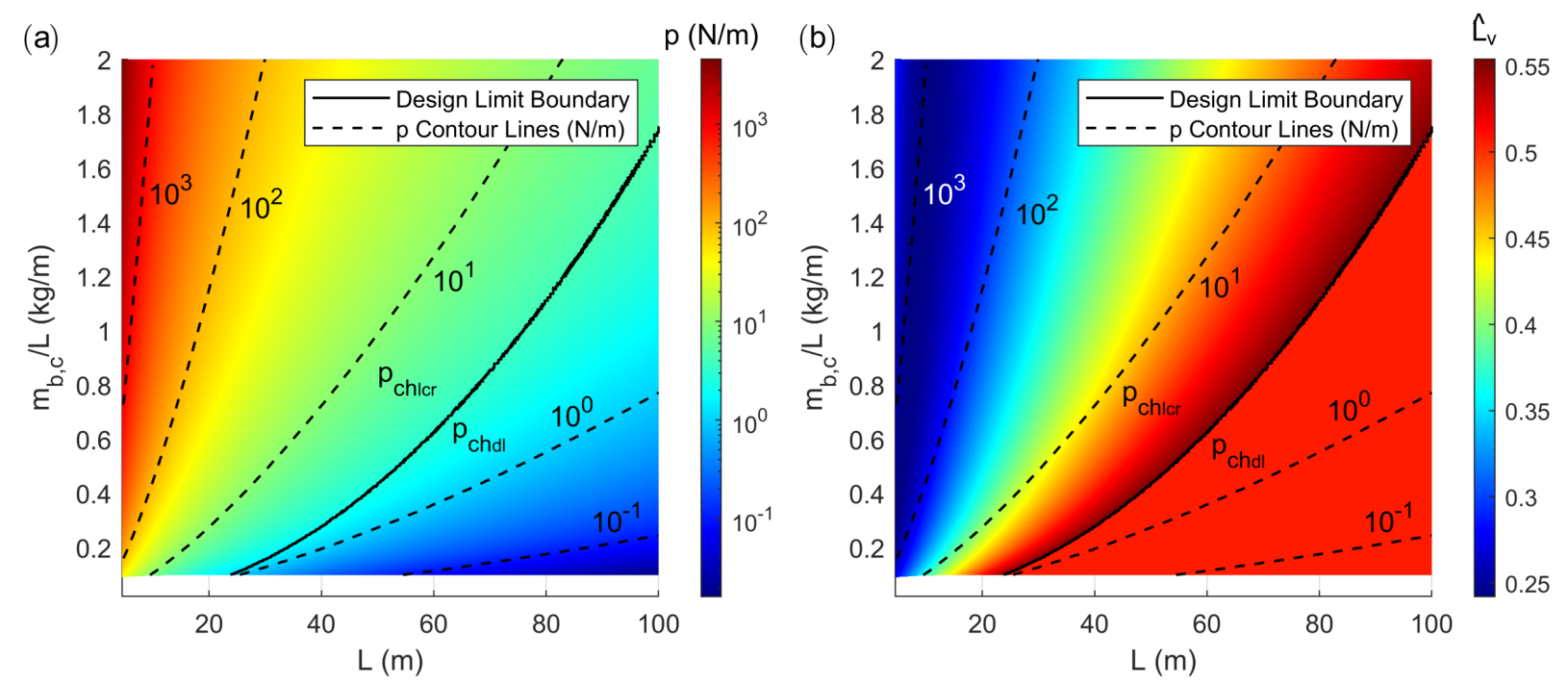

Fig. 8 (a) Maximum load carried by optimal designs and (b) corresponding $\hat{L}_{v}$, as a function of $L$ and $\frac{m_{b, c}}{L}$. Solid lines mark the transitions between different limiting conditions. Dashed lines mark order-of-magnitude variations of the maximum load limit.

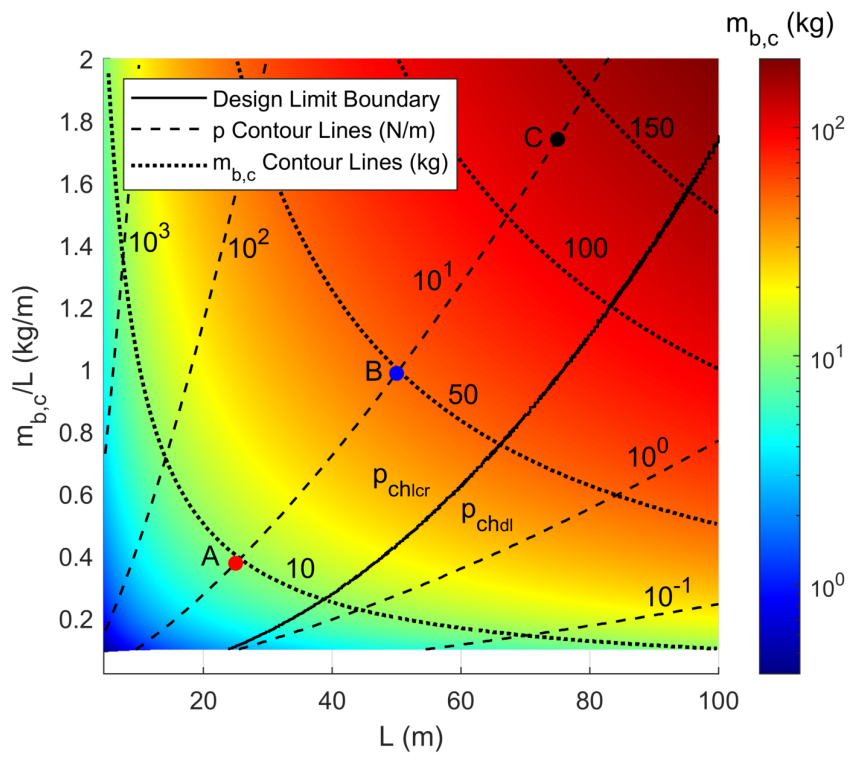

Fig. 9 Total mass of optimal designs as a function of $L$ and $\frac{m_{b, c}}{L}$. Solid lines mark the transitions between different limiting conditions. Dashed lines mark order-of-magnitude variations of the maximum load limit. Dotted lines mark variations of total mass. Optimal cable-stayed designs A, B, and C are shown in Fig. 10 .

For the nominal material properties, thickness to radius ratio $\frac{t_{c}}{r_{c}}=0.0075$, and the specified length and linear mass density ranges in Figs. 8 and 9 , the cable-stayed architecture remains optimal over the full range. Fig. 8 (a) shows that the optimal cable-stayed designs with lower $L$ and higher $\frac{m_{b, c}}{L}$ will fail first through local shell buckling of the horizontal beam-column, which corresponds to larger loads $p_{c_{\text {lim }}}$. When the length is increased and the linear mass density is decreased, the optimal designs transition to being limited by excessive deflection and the maximum loads $p_{c_{\text {lim }}}$ are lowered.

Fig. 9 shows the variation of the total mass for the optimal designs with respect to the length and the maximum 
load limit. It shows that the linear mass density and therefore the mass must be increased to maintain a constant load limit while increasing $L$. Fig. 8 (b) shows that $\hat{L}_{v}$ has a similar variation with respect to $L$ and $\frac{m_{b, c}}{L}$ as the maximum load limit in the region where the optimal cable-stayed designs will fail through local shell buckling of the horizontal beam-column. However, $\hat{L}_{v}$ remains constant at 0.505 when the limiting condition is excessive deflection.

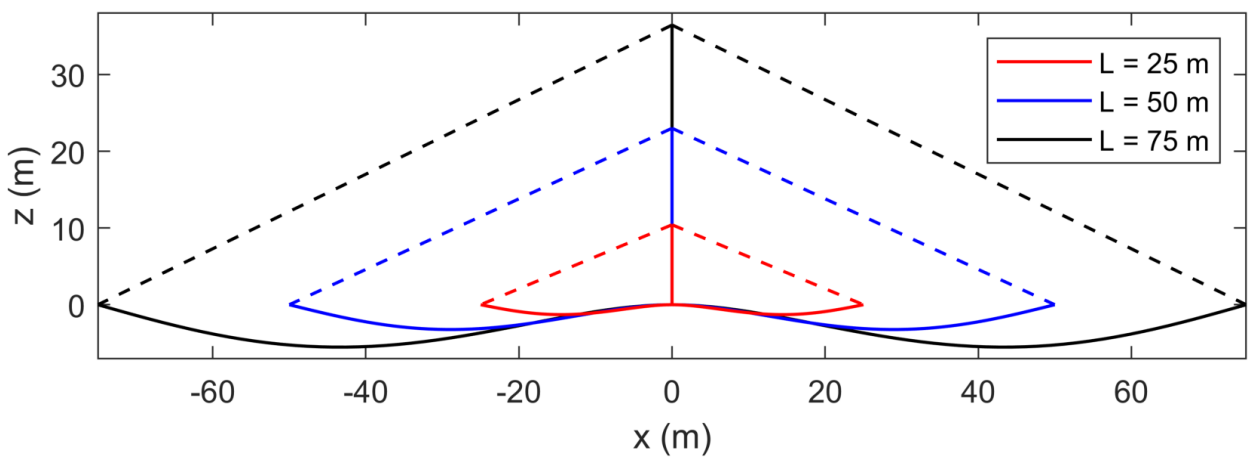

Fig. 10 Deflection of optimal cable-stayed designs A, B, and C from Fig. 9 for a load limit of 10 N/m.

Table 2 Geometric parameters, mass, and maximum deflection of the optimal cable-stayed designs.

\begin{tabular}{ccccccc}
\hline Design & $L(\mathrm{~m})$ & $L_{v}(\mathrm{~m})$ & $r_{c}(\mathrm{~mm})$ & $t_{c}(\mathrm{~mm})$ & $m_{c}(\mathrm{~kg})$ & $w_{c h_{\max }}(\mathrm{m})$ \\
\hline A & 25 & 10.4 & 61.3 & 0.459 & 9.40 & -1.29 \\
B & 50 & 23.0 & 98.4 & 0.738 & 49.4 & -3.24 \\
C & 75 & 36.5 & 130 & 0.974 & 131 & -5.53 \\
\hline
\end{tabular}

Three specific optimal designs have been chosen in Fig. 9 and labelled as A, B, and C. Suppose that the load carrying requirement is $p=10 \mathrm{~N} / \mathrm{m}$ and hence select designs $\mathrm{A}, \mathrm{B}$, and $\mathrm{C}$ with $L=25,50$, and $75 \mathrm{~m}$ along the $p=10$ $\mathrm{N} / \mathrm{m}$ contour line in Fig. 9. The figure shows that the required linear mass density will be $0.376,0.987$, and $1.74 \mathrm{~kg} / \mathrm{m}$, respectively. It also shows that these designs will be limited by local shell buckling of the horizontal beam-column rather than excessive deflection. Fig. 8(b) provides the corresponding vertical column length for these optimal designs, $\hat{L}_{v}=0.417,0.460$, and 0.486 , respectively. Their deflected shapes under $p=10 \mathrm{~N} / \mathrm{m}$ have been plotted in Fig. 10 and Table 2 lists the corresponding horizontal length, vertical column length, tube radius, thickness, mass, and maximum deflection. To maintain the $p=10 \mathrm{~N} / \mathrm{m}$ load carrying capability, the linear mass density must increase with larger lengths and this is indicated by the increasing radius and thickness from designs A through $\mathrm{C}$. The maximum deflections are all confirmed to be sufficiently small relative to length of each design.

\section{Conclusions}

An analytical framework has been presented for evaluating the load carrying capability of the cable-stayed architecture relative to an equivalent bending architecture. Considering structures made of the same material, the maximum load that can be carried by optimal designs of the two architectures have been compared for equal mass and maximum deflection. Global buckling, local shell buckling, material failure, and excessive deflection of the main structural members were considered in the comparison.

An initial design case has shown that the cable-stayed architecture will either fail through local buckling of the horizontal beam-column or global buckling of the vertical column, depending on its vertical column length. In contrast, the bending architecture is limited by excessive deflection or will fail through local buckling. When the load limits of optimal designs are compared, the cable-stayed architecture carries the highest load and the corresponding design is the optimum for the geometric and material parameters chosen in the present study.

This comparison has been extended to a wide range of lengths and the present analysis has shown that the cable-stayed architecture remains optimal, with the maximum loads corresponding to local shell buckling of the horizontal beam-column for shorter lengths and excessive deflection for larger lengths. A study of the optimal designs 
for various linear mass densities has demonstrated that the cable-stayed architecture remain optimal across the full range considered and its mass scales with length to carry a constant load magnitude. Overall, the cable-stayed architecture has been shown to be more efficient at withstanding external loads than the bending architecture.

\section{Acknowledgments}

The authors are grateful to Dr. Martin Mikulas of the National Institute of Aerospace for sharing his experience and for insightful discussions. Financial support from the Space Solar Power Project at the California Institute of Technology is gratefully acknowledged.

\section{References}

[1] Jones, P. A., and Spence, B. R., "Spacecraft solar array technology trends," IEEE Aerospace and Electronic Systems Magazine, Vol. 26, No. 8, 2011, pp. 17-28.

[2] Fu, B., Sperber, E., and Eke, F., "Solar sail technology—a state of the art review," Progress in Aerospace Sciences, Vol. 86, 2016, pp. 1-19.

[3] Sokolowski, W. M., and Tan, S. C., "Advanced self-deployable structures for space applications," Journal of Spacecraft and Rockets, Vol. 44, No. 4, 2007, pp. 750-754.

[4] Murphey, T., "Historical perspectives on the development of deployable reflectors," 50th AIAA/ASME/ASCE/AHS/ASC Structures, Structural Dynamics, and Materials Conference, 2009, p. 2605.

[5] Feria, V., Lou, M., Huang, J., and Speer, S., "Lightweight deployable space radar arrays," 39th AIAA/ASME/ASCE/AHS/ASC Structures, Structural Dynamics, and Materials Conference and Exhibit, 1998, p. 1933.

[6] Thomas, V. C., Makowski, J. M., Brown, G. M., McCarthy, J. F., Bruno, D., Cardoso, J. C., Chiville, W. M., Meyer, T. F., Nelson, K. E., Pavri, B. E., et al., “The Dawn spacecraft,” Space Science Reviews, Vol. 163, 2011, pp. 175-249.

[7] Matousek, S., “The Juno new frontiers mission,” Acta Astronautica, Vol. 61, No. 10, 2007, pp. 932-939.

[8] Banik, J. A., and Maji, A. K., "Structural scaling parameters for rectangular flexible blanket solar arrays," Journal of Spacecraft and Rockets, Vol. 53, No. 5, 2016, pp. 936-951.

[9] Greschik, G., and Mikulas, M., "Design study of a square solar sail architecture," Journal of Spacecraft and Rockets, Vol. 39, No. 5, 2002, pp. 653-661.

[10] Howson, W. P., and Williams, F. W., "A parametric study of the initial buckling of stayed columns," Proceedings of the Institution of Civil Engineers, Vol. 69, No. 2, 1980, pp. 261-279.

[11] Heard Jr, W. L., Bush, H. G., Walz, J. E., and Rehder, J. J., "Structural sizing considerations for large space platforms," Journal of Spacecraft and Rockets, Vol. 18, No. 6, 1981, pp. 556-564.

[12] Hedgepeth, J., "Critical requirements for the design of large space structures," 2nd Conference on Large Space Platforms: Toward Permanent Manned Occupancy in Space, 1981, p. 443.

[13] Belvin, W. K., "Analytical and experimental vibration and buckling characteristics of a pretensioned stayed column," Journal of Spacecraft and Rockets, Vol. 21, No. 5, 1984, pp. 456-462.

[14] Jones, T., Watson, J., Mikulas, M., and Bart-Smith, H., "Design and analysis of tension-aligned large aperture sensorcraft," 49th AIAA/ASME/ASCE/AHS/ASC Structures, Structural Dynamics, and Materials Conference, 2008, p. 1775.

[15] Lane, S. A., Murphey, T. W., and Zatman, M., "Overview of the innovative space-based radar antenna technology program," Journal of Spacecraft and Rockets, Vol. 48, No. 1, 2011, pp. 135-145.

[16] Timoshenko, S. P., and Gere, J. M., Theory of elastic stability, Dover Publications, 1961.

[17] Brush, D. O., and Almroth, B. O., Buckling of bars, plates, and shells, McGraw-Hill, New York, 1975.

[18] Peterson, J. P., Seide, P., and Weingarten, V. I., "Buckling of Thin-Walled Circular Cylinders,” NASA SP-8007, 1965. 